\title{
Poids des chevreaux de la population locale Sud tunisienne dans des conditions d'aridité : impacts lors des procédures de sélection
}

\author{
Ahlem Atoui ${ }^{1 *}$ María Jésús Carabaño ${ }^{2}$ \\ Moldi Abdennebi ${ }^{1}$ Sghaier Najari ${ }^{1}$
}

\begin{abstract}
Mots-clés
Caprin, système d'élevage, croissance, sélection, facteur du milieu, Tunisie
\end{abstract}

\footnotetext{
Submitted: 30 September 2020

Accepted: 26 April 2021

Published: 30 June 2021

DOI: $10.19182 /$ remvt.36375
}

\begin{abstract}
Résumé
La nature et la distribution des phénotypes quantitatifs représentent une connaissance de base pour l'application des méthodologies génétiques dans les plans de sélection des animaux domestiques. Afin d'illustrer l'action de facteurs non génétiques d'aridité sur la distribution phénotypique des performances individuelles de la croissance des chevreaux de la population locale élevés dans des conditions restrictives et irrégulières, 945 chevreaux issus de 285 chèvres et 19 boucs, nés durant la période 1998-2014, ont été étudiés par l'analyse des distributions des poids jusqu'à 150 jours d'âge. La dispersion des poids représentait une expression collective des génotypes des chevreaux dans les conditions de l'étude avec des variations dues à la fois aux effets génétiques et environnementaux. L'hypothèse de la normalité n'a été vérifiée qu'aux âges de 10 jours et 30 jours quand les chevreaux étaient surtout nourris sous la mère et n'étaient pas directement dépendants des ressources alimentaires du parcours aride. Durant une année défavorable, les performances enregistrées ont suivi une distribution particulière expliquée par le fait que les chevreaux performants ont été incapables d'extérioriser leur supériorité. Par conséquent, le milieu aride a réduit les performances de la population caprine locale. En outre, il a limité l'expression de quelques génotypes fortement productifs. Cette spécificité de l'action du milieu aride incite à un approfondissement indispensable pour ajuster les méthodes de sélection aux conditions des milieux restrictifs et des groupes génétiques adaptés.
\end{abstract}

- Comment citer cet article : Atoui A., Carabaño M.J., Abdennebi M., Najari S., 2021. Weights of caprine kids of the local Southern Tunisian population under arid conditions: Impacts during selection process. Rev. Elev. Med. Vet. Pays Trop., 74 (2): 121-126, doi: 10.19182/remvt.36375

\section{INTRODUCTION}

La croissance des chevreaux est l'un des caractères les plus considérés dans les schémas d'amélioration génétique des caprins, surtout au sein des modes d'élevage lactant. A l'instar des phénotypes quantitatifs des animaux domestiques, un certain nombre de facteurs non génétiques affectent ces caractères de croissance et masquent la connaissance directe du vrai potentiel génétique de l'animal. Par conséquent, la mise en évidence des effets de l'action du milieu dans lequel le génotype a été exprimé est essentielle pour obtenir des

1. Laboratoire d'élevage et de la faune sauvage, Institut des régions arides, Médenine, Université de Gabès, Tunisie

2. Département d'amélioration génétique animale, INIA, 28040 Madrid, Espagne.

* Auteur pour la correspondance

Email : ahlematoui@gmail.com estimations précises des paramètres et des prédictions correctes des valeurs génétiques (Atoui et al., 2019 ; Najari, 2005).

En outre, l'identification des performances réelles de production de la chèvre locale au niveau de la productivité numérique et de la croissance, ainsi que leurs modes de variation en fonction des facteurs de production sont un préalable. La modélisation de ces performances est indispensable pour l'élaboration des procédures d'évaluation des géniteurs (Atoui et al., 2019). En effet, le principal objectif des index de sélection est de prédire les potentialités génétiques héritables des géniteurs potentiels via la correction par les effets des facteurs non génétiques. Pour cette raison l'établissement des principales composantes des phénotypes de production est indispensable pour l'élaboration des méthodologies d'évaluation qui prédisent, au mieux, les valeurs génétiques des animaux exprimées dans un contexte de vie et de production déterminé (Zhang et al., 2008 ; Rashidi et al., 2015). Les procédures statistiques de correction des phénotypes par les effets du milieu se basent sur l'hypothèse de la normalité des distributions observées et de la libre aptitude d'expression des génotypes. 
De fait, l'évolution des méthodologies de sélection a été réalisée dans des conditions assez favorables à la manifestation des potentialités héritables, ce qui n'est pas potentiellement le cas dans des environnements arides avec des conditions restrictives et irrégulières (Najari, 2006). D'où la nécessité de l'identification de la nature de l'impact du milieu aride sur la structure statistique de base des performances réalisées afin d'assurer les conditions d'application des procédures et des méthodologies de sélection (Najari, 2005).

La présente étude repose sur l'analyse des performances individuelles de chevreaux de la population locale élevés en conditions d'aridité. Elle vise à montrer l'action de facteurs d'aridité sur la distribution des poids des caprins, qui sont fréquemment considérés dans les critères de sélection des cheptels lactants. L'étude des distributions est indispensable pour orienter la correction des effets de l'environnement et pour mieux maitriser les sources de variation des performances de façon à déterminer par la suite la nature de la variabilité, et surtout ses spécificités, en fonction des conditions environnementales.

\section{MATERIEL ET METHODES}

\section{Zone d'étude}

La région d'étude, située entre la chaîne de Matmata à l'ouest et la Méditerranée à l'est, est soumise à un climat méditerranéen continental aride. Elle est caractérisée par un climat aride, avec une pluviométrie irrégulière, inférieure à $200 \mathrm{~mm}$ par an en moyenne, et des vents dominants d'ouest, de nord-est et de sud-est de novembre à avril. La température moyenne annuelle est de $20,2^{\circ} \mathrm{C}$, avec la moyenne la plus basse $\left(13,2^{\circ} \mathrm{C}\right)$ en décembre et la plus haute $\left(30,8^{\circ} \mathrm{C}\right)$ en juillet. L'été est normalement la saison la plus chaude et la plus sèche avec une température maximale de $47^{\circ} \mathrm{C}$ (Atoui et al., 2019).

\section{Population caprine locale}

La population caprine locale est très hétérogène sur le plan génétique et morphologique. Najari (2005) rapporte : «Le cheptel caprin local constitue une population animale rustique à large variabilité au niveau de la morphologie comme au niveau des performances. Cette population regroupe plusieurs types pigmentaires à cause de l'intégration » de plusieurs races ou groupes génétiques « dans ses origines, à l'instar de la race Nubienne diluée dans la population locale». Le pelage noir constitue le type dominant chez la chèvre locale avec une fréquence supérieure à $60 \%$; la fréquence des robes pie est environ de $20 \%$. Le même auteur signale la présence d'autres couleurs comme le rougeâtre ou le blanchâtre (figure 1). Toutefois, une variabilité plus marquée a été observée au niveau de la couleur de la tête avec des motifs incluant le noir, le blanc et le roux (Najari, 2005). Najari (2006) suggère une relation entre la morphologie de la chèvre locale et la géographie de la zone. Ainsi, la morphologie de la chèvre élevée dans les régions côtières se distingue de celle observée dans les zones de montagne ou dans les plaines. Le dénombrement effectué par les auteurs sur un effectif de 6500 chèvres adultes montre que le type noir est présent avec la même fréquence dans les différentes zones. Le type nommé Hawa qui présente des traits roux ou blancs sur les joues, les oreilles et le ventre est dominant dans les élevages des steppes, alors que le type dit Gharra qui montre des taches blanches sur le front est présent à une fréquence de $21 \%$ et $30 \%$ respectivement dans les régions côtières et montagneuses. Ce sont les troupeaux de la plaine et de la côte qui montrent la variabilité la plus importante.

La chèvre locale est par ailleurs connue pour sa petite taille à l'instar de la plupart des races et des populations adaptées aux zones chaudes et désertiques. A cet égard, la hauteur moyenne au garrot est de 73 $\pm 3,80 \mathrm{~cm}$ chez les boucs et de seulement $60 \pm 2,44 \mathrm{~cm}$ chez les chèvres. Leurs poids adultes moyens respectifs sont de $35 \pm 6,60 \mathrm{~kg}$ et $24 \pm 4,55 \mathrm{~kg}$ (observations de El Ouni et Najari).

\section{Troupeau expérimental et suivi}

Les informations relatives aux phénotypes et aux facteurs de production comme les pesées des chevreaux dès la naissance et jusqu'à l'âge de six mois ont été collectées chez les caprins du troupeau expérimental de l'Institut des régions arides de Médenine (IRA), situé à 22 kilomètres de la ville de Médenine, au sud-est de la Tunisie. La base des données issues du suivi périodique de 1984 à 2014 regroupe les poids et les informations relatifs à 945 chevreaux issus de 285 chèvres et 19 boucs. Les chevreaux étaient d'abord nourris sous la mère, à un mois d'âge ils commençaient à consommer de l'aliment solide et le sevrage était pratiqué en juin. Ils étaient pesés tous les 15 jours.

\section{Analyse statistique et estimation des variables}

Pour chaque chevreau, l'estimation du poids aux âges types (PAT) a été obtenue par interpolation linéaire lorsque deux pesées, le poids de naissance non compté, encadraient l'âge type voulu, en appliquant la formule suivante :

$$
P A T_{i}=P_{n}+\frac{[P n+1-P n]}{[D n+1-D n]} \times\left[i-A_{n}\right]
$$

où $i$ est l'âge type du chevreau, $P_{n}, P_{n+1}, D_{n}$ et $D_{n+1}$ représentent respectivement les poids et les dates de contrôle de croissance $n$ et $n+l$, et $A_{n}$ est l'âge à la pesée $n\left(\operatorname{avec} A_{n}<i<A_{n+1}\right)$. Dans les cas où il ne peut y avoir interpolation, le calcul peut être réalisé par extrapolation linéaire inférieure (où $i<A_{n}<A_{n+1}$ ) ou extrapolation linéaire supérieure (où $A_{n}<A_{n+1}<i$ ). Les caractères de $P A T$ considérés pour cette étude ont été les poids à la naissance, à 10,30,60,90, 120 et 150 jours. Des tests de normalité ont été appliqués afin d'évaluer le modèle de distribution statistique des performances étudiées.

Les performances étudiées étaient des phénotypes quantitatifs qui synthétisaient les effets environnementaux et l'expression du génotype animal. Il est indispensable, pour l'évaluation de la valeur génétique héritable des chevreaux, de déterminer les sources de variation des performances de croissance qui sont dues à des facteurs non transmissibles. L'étude de ces sources de variation a nécessité le recours à une décomposition de la variance (GLM, modèle linéaire généralisé) avec le logiciel SPSS.20 pour illustrer la nature statistique de l'action

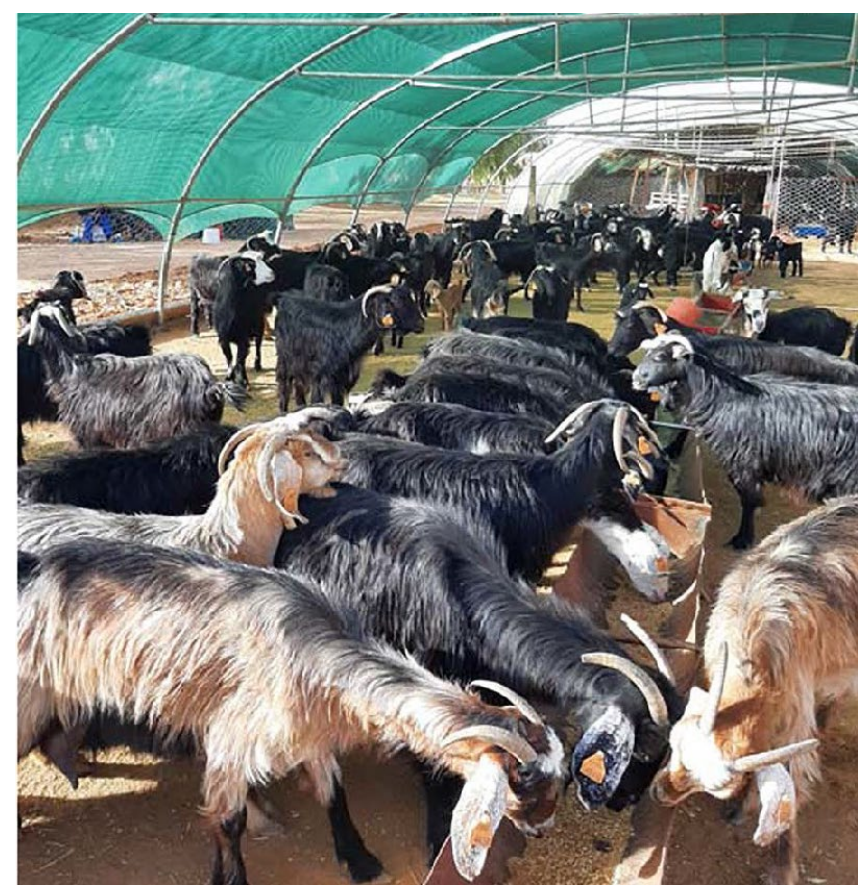

Figure 1 : Population caprine locale tunisienne /// Local Tunisian goat population 
des différents facteurs fixes inclus dans le modèle de base. Le modèle de décomposition de la variance a été le suivant :

$Y_{i m}=\mu+A n_{i}+S_{m}++e_{i m}$

où $Y_{i m}$ est la performance à analyser (poids en $\mathrm{kg}$ ), $\mu$ la moyenne générale, $A n_{i}$ l'effet de l'année de naissance ( $\left.i=1998,1999 . . .2014\right)$, $S_{m}$ l'effet du sexe ( $m=$ mâle ou femelle) et $e_{i m}$ l'erreur résiduelle. Ensuite nous avons réalisé le test de comparaison des moyennes de Student Newman et Keuls $(\alpha=0,05)$ afin de comparer les moyennes des modalités de chaque facteur de variation qui illustre un effet au moins significatif ( $p<0,05$ ou $p<0,01)$.

\section{RESULTATS}

\section{Dispersion des performances de croissance}

La figure 2 illustre l'évolution des poids des chevreaux de la population caprine locale en fonction de l'âge. Cette simple présentation des données de pesées des chevreaux témoigne d'une variation considérable au niveau des phénotypes individuels. La dispersion des poids représente une expression collective des génotypes des chevreaux dans les conditions observées durant les 16 campagnes de suivi. Le problème ne réside pas dans la variabilité importante qui peut être exploitée par sélection mais plutôt dans sa nature qui rend difficile la modélisation des performances. Ce type de dispersion est typique des performances des animaux réalisées dans des conditions difficiles et variables (Najari, 2006).

Suite à l'estimation des poids aux âges types individuels, des tests de normalité ont été appliqués afin d'évaluer la nature de la distribution statistique de ces performances étudiées ou phénotypes quantitatifs. En effet, certaines spécificités, en étroite relation avec la génétique quantitative et la sélection des animaux domestiques, ont été signalées par Atoui et al. (2019) dans des cas similaires. La distribution de

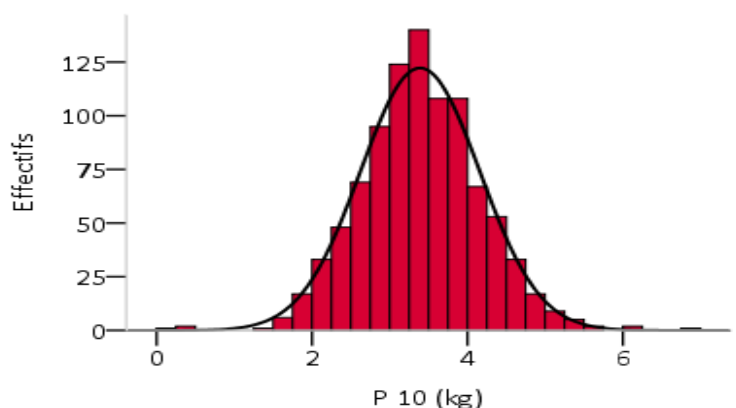

certaines performances des chevreaux peut ne pas vérifier les hypothèses de la normalité en vertu des conditions des régions arides et de leurs effets sur l'expression des génotypes (Najari, 2005). Ce problème manifeste une difficulté d'expression des génotypes les plus performants dans des conditions restrictives. L'hypothèse de la normalité n'a été vérifiée qu'à l'âge de 10 et 30 jours (tableau I, figure 3), âges où les jeunes sont essentiellement nourris au lait maternel et ne sont pas directement dépendants des ressources alimentaires du parcours (Goffinet et Elsen, 1984 ; Dekkers, 2002).

\section{Paramètres statistiques et marges de variation des poids aux âges types}

Le tableau I montre une description statistique des poids aux âges types des chevreaux locaux élevés dans des conditions arides ainsi que les résultats des tests de normalité de ces phénotypes quantitatifs et critères potentiels de sélection. Les poids moyens des chevreaux étaient de

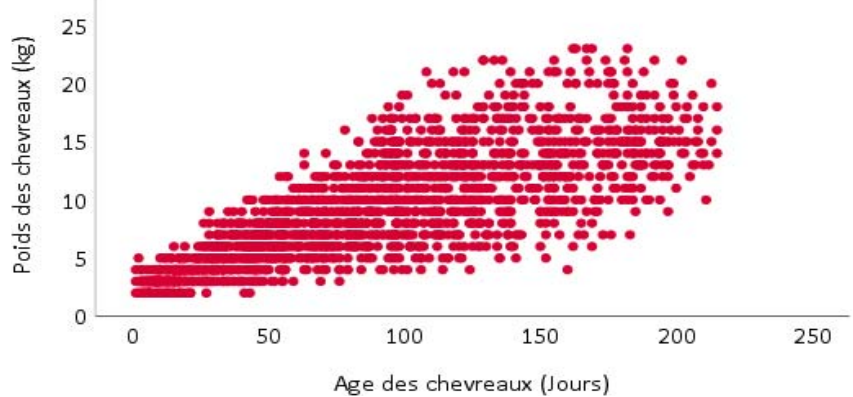

Figure 2 : Répartition des poids de 945 chevreaux locaux tunisiens en fonction de l'âge /// Weight distribution of 945 local Tunisian caprine kids according to age

Figure 3 : Répartition des poids des chevreaux locaux tunisiens à 10 et à 30 jours /// Weight distribution of local Tunisian caprine kids at 10 and 30 days

Tableau I : Paramètres statistiques des poids aux âges types (en jours) de 945 chevreaux locaux tunisiens /// Statistical parameters of weights at typical ages (in days) of 945 local Tunisian caprine kids

\begin{tabular}{|c|c|c|c|c|c|c|c|}
\hline Paramètre & Naissance & 10 jrs & 30 jrs & 60 jrs & 90 jrs & 120 jrs & $150 \mathrm{jrs}$ \\
\hline Effectif & 945 & 945 & 945 & 945 & 945 & 945 & 945 \\
\hline Moyenne (kg) & 2,50 & 3,10 & 4,80 & 7,20 & 9,80 & 12,20 & 12,90 \\
\hline Ecart-type (kg) & 0,57 & 0,66 & 1,18 & 1,87 & 2,72 & 4,11 & 3,84 \\
\hline Minimum (kg) & 1,12 & 1,2 & 2,32 & 3,34 & 4,34 & 6,03 & 6,23 \\
\hline Maximum (kg) & 3,95 & 4,12 & 5,45 & 8,64 & 11,12 & 13,33 & 16,36 \\
\hline Coefficient de variation (\%) & 22,90 & 21,30 & 24,60 & 26,00 & 27,80 & 33,70 & 29,80 \\
\hline Coefficient de Kolmogorov & 0,001 & 0,131 & 0,200 & 0,021 & 0,021 & 0,001 & 0,011 \\
\hline Coefficient de ShapiroWilk & 0,022 & 0,220 & 0,391 & 0,033 & 0,042 & 0,001 & 0,010 \\
\hline Test de normalité Kolmogorov & Rejeté & Accepté & Accepté & Rejeté & Rejeté & Rejeté & Rejeté \\
\hline Test de normalité Shapiro-Wilk & Rejeté & Accepté & Accepté & Rejeté & Rejeté & Rejeté & Rejeté \\
\hline
\end{tabular}


2,50 kg, 3,10 kg, 4,80 kg, 7,20 kg, 9,80 kg, 12,20 kg et 12,90 kg, respectivement à la naissance, et à 10 jours, 30 jours, 60 jours, 90 jours, 120 jours et 150 jours. Ces performances de croissance relativement faibles illustrent les aptitudes réelles de production de la population locale ; les écarts-types élevés traduisent la variabilité importante des caractères de croissance de la population caprine locale.

\section{Sources de variation des performances de la croissance}

L'analyse de la variance a permis d'identifier les principales sources de variation de ces performances à des âges prédéfinis (tableau II). Le coefficient de détermination $\left(\mathrm{R}^{2}\right)$ a varié de 0,63 à 0,81 et il a évolué sensiblement avec l'âge des chevreaux. Un coefficient de détermination relativement élevé atteste de l'importance des effets fixes inclus dans le modèle de décomposition de la variance adoptée. La décomposition de la variance a montré que les performances de croissance des chevreaux locaux étaient fortement tributaires des conditions du milieu. Les résultats soulignent l'importance de l'effet de la campagne sur les performances à tous les âges des chevreaux. Ces résultats ont bien reflété l'importance des effets de l'irrégularité des climats arides, d'autant que la période de suivi était assez longue pour illustrer des conditions d'élevage très diverses. Par ailleurs, le sexe a affecté significativement la plupart des poids aux âges types. L'effet de l'interaction année $\times$ sexe montre que les impacts des facteurs climatiques peuvent être différemment ressentis chez les chevrettes et les chevreaux (tableau II).

\section{Distribution des poids aux âges types de 10 jours et 30 jours}

Les tests de normalité appliqués sur les performances de 945 chevreaux ont rejeté l'hypothèse de la normalité pour la plupart des performances, surtout celles réalisées après l'âge d'un mois (tableau I). Ces résultats ont concerné les performances réalisées durant 17 campagnes d'élevage durant lesquelles les conditions ont varié de très favorables à très mauvaises. La distribution des poids des chevreaux à 30 jours a changé considérablement d'une année à l'autre pour refléter l'expression des génotypes dans des conditions de pluviométrie différentes (figure 4). La pluviométrie annuelle était de $316 \mathrm{~mm}$ en 1999 et de $41 \mathrm{~mm}$ en 2011. En 1999, les performances enregistrées ont suivi une distribution normale confirmée par les tests de normalité (Kolmogorov et Shapiro-Wilk). En 2011 (année sèche), elles ont présenté une distribution particulière expliquée par le fait que les chevreaux performants ont été incapables d'extérioriser leur supériorité.

\section{DISCUSSION}

\section{Dispersion des performances de croissance}

La visualisation de la dispersion des performances brutes met en évidence certains aspects relatifs à la nature de la distribution de l'information observée (ElOuni et Najari, 2019 ; Najari, 2006). L'importance de cette étape se manifeste notamment lors de l'étude des bases de données collectées dans des conditions difficiles ou non classiques, et durant une période assez longue (Falconer, 1989 ; Goffinet et Elsen, 1984).

Au sujet de la dispersion des poids des chevreaux dans les conditions observées durant les 17 campagnes de suivi, une grande variabilité est, certes, toujours souhaitable dans les programmes d'amélioration génétique par la sélection. Cependant, dans le cas présent cette grande dispersion a plutôt manifesté un effet non génétique attribuable à la nature du milieu aride qui pouvait empêcher l'application des outils méthodologiques de la génétique quantitative lors de la distinction entre effets héritables et effets non génétiques.

La variabilité la plus importante au niveau de la dispersion a été observée pour les valeurs élevées ; les poids les plus légers ont été plus homogènes. Quant aux faibles performances, il s'agissait d'un comportement général chez la population rustique en conditions d'élevage défavorables. L'amplitude de la dispersion a augmenté avec l'âge : dès trois mois, la

Tableau II : Analyse de la variance des poids aux âges types de 945 chevreaux locaux tunisiens /// Analysis of variance of weights at standard ages of 945 local Tunisian caprine kids

\begin{tabular}{|c|c|c|c|c|c|c|c|c|}
\hline \multirow[t]{2}{*}{ Facteur de variation } & \multirow[t]{2}{*}{ DDL } & \multicolumn{7}{|c|}{ Poids (kg) aux âges types (jours) } \\
\hline & & $1 \mathrm{jr}$ & 10 jrs & 30 jrs & 60 jrs & 90 jrs & 120 jrs & 150 jrs \\
\hline Année de naissance & 15 & $* *$ & $* *$ & $* *$ & $* *$ & $* *$ & $* *$ & $* *$ \\
\hline Sexe & 1 & $* *$ & $* *$ & $* *$ & $* *$ & $* *$ & $* *$ & $* *$ \\
\hline Année $\times$ sexe & 15 & $*$ & * & * & NS & * & NS & NS \\
\hline R2 & & 0,71 & 0,63 & 0,63 & 0,68 & 0,73 & 0,73 & 0,81 \\
\hline
\end{tabular}

$* \mathrm{p}<0,05 ; * * \mathrm{p}<0,01 ; \mathrm{NS}: \mathrm{p}>0,05 ; \mathrm{R}^{2}:$ coefficient de détermination ///*p<0.05; ** $p<0.01 ;$ NS: $p>0.05 ; R^{2}:$ coefficient of determination
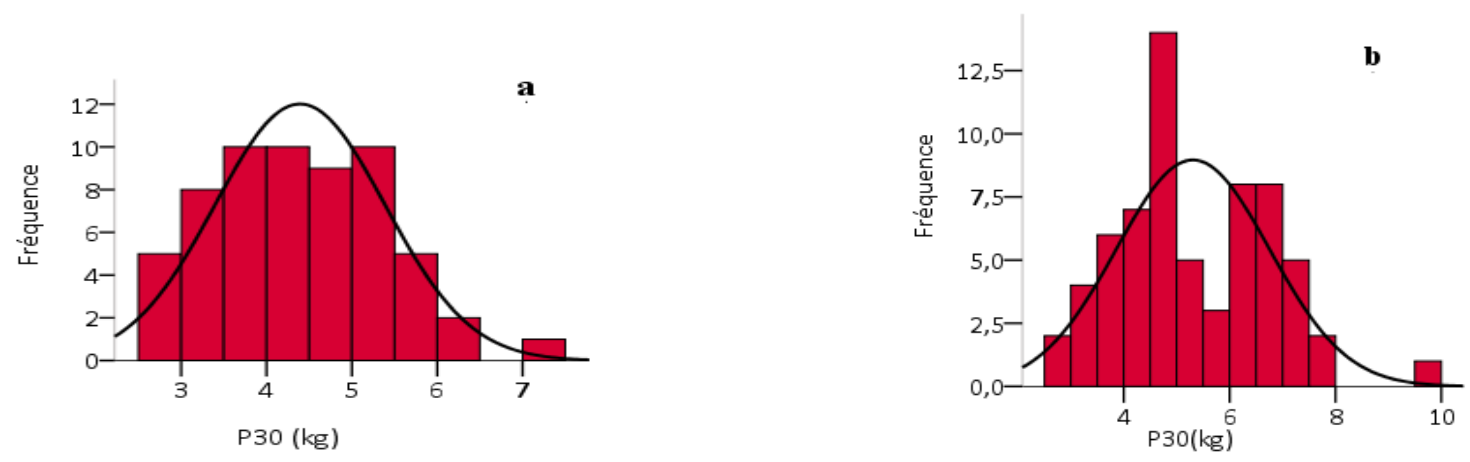

Figure 4 : Répartition des poids des chevreaux locaux tunisiens à 30 jours ; a) au cours d'une année pluvieuse (1999) ; b )au cours d'une année sèche (2011) /// Weight distribution of local Tunisian caprine kids at 30 days; a) during a rainy year (1999); b) during a dry year (2011) 
marge de variation des poids est devenue considérable et la différence entre les maximums et les minimums a dépassé 15 kilogrammes. La dispersion des performances permet à elle seule d'estimer les problèmes qui peuvent se poser lors de l'application des procédures de prédiction des valeurs génétiques ou d'estimation des paramètres génétiques des candidats à la sélection. Par ailleurs, l'ajustement de la courbe de la cinétique de la croissance en fonction de l'âge pourrait rencontrer des difficultés de convergence avec de telles dispersions.

\section{Paramètres statistiques et marges de variation des poids aux âges types}

Dans les programmes de sélection des cheptels lactants, les critères de sélection comprennent généralement les poids aux âges types (naissance, 10, 30, 60, 90 jours...) et les gains moyens quotidiens, ce qui impose l'étude de leur distribution. Nos résultats ont montré l'importance de la marge de variation des phénotypes réalisés durant 17 campagnes de suivi dans des conditions arides très irrégulières. Par exemple, le maximum du poids à la naissance $(3,95 \mathrm{~kg})$ a été supérieur à la moyenne des poids à 10 jours $(3,1 \mathrm{~kg})$ et au minimum des poids à 60 jours $(3,34 \mathrm{~kg})$. Les résultats montrent que la période de croissance peut être scindée en deux phases qui tiennent compte de la variabilité observée des phénotypes. Dans la phase qui va de la naissance à trois mois, les différences entre les performances individuelles sont aisément observables ce qui favorise la mise au point des procédures de sélection classiques. Dans la deuxième phase, lorsque le chevreau est âgé de plus de trois mois, l'aridité du milieu empêche l'expression complète des génotypes, surtout des meilleurs ; ainsi tous les candidats ne réalisent que des performances comparables imposées par la rareté des ressources.

\section{Sources de variation des performances de croissance}

L'année de naissance s'est révélée avoir un effet très significatif $(\mathrm{p}<0,01)$ sur le poids des chevreaux dès la naissance jusqu'à 150 jours d'âge. Cette différence entre les années est attribuée principalement aux variations pluviométriques, surtout pour un élevage qui repose pour une grande partie de son alimentation sur le parcours. L'effet très significatif de l'année de naissance sur les performances de croissance a également été rapporté par Goffinet et Elsen (1984). Le climat méditerranéen se caractérise par des grandes fluctuations climatiques inter- et intraannées qui affectent les ressources pastorales, et ainsi les aptitudes productives des animaux. En effet, l'état des parcours affecte directement la croissance à travers la disponibilité des fourrages et indirectement par le biais de la production laitière de la mère lactante; notons que dans le système d'élevage local du Sud tunisien les chevreaux sont sous la mère jusqu'à l'âge du sevrage estimé à six mois. Ces fluctuations s'observent plus fortement dans le cas des élevages extensifs et surtout lorsque la base de données comporte plusieurs années de suivi. L'oscillation des performances, attribuée à l'année, caractérise non seulement l'environnement naturel aride et ses irrégularités, mais aussi les variations d'autres facteurs climatiques (température, humidité de l'air...) ainsi que les variations de la conduite et de la disponibilité en aliments de complémentation. Tous ces facteurs peuvent agir positivement ou négativement sur l'expression des génotypes des chevreaux (Najari, 2005).

Le sexe des chevreaux a présenté un effet significatif sur les performances étudiées. Dès la naissance et jusqu'à l'âge de 150 jours, les mâles ont été significativement plus lourds et ont gagné du poids plus rapidement. L'écart de poids entre les mâles et les femelles s'est rallongé en faveur des mâles à mesure que les animaux devenaient plus âgés, avec une différence de $0,29 \mathrm{~kg}$ à la naissance et de 1,59 kg à cinq mois. Ces avantages de croissance des chevreaux mâles enregistrés dans notre étude étaient comparables à ceux relevés pour d'autres races caprines (Goffinet et Elsen, 1984). Toutefois, Rashidi et al. (2015) rapportent que le sexe des chevreaux n'a pas d'effet sur le poids corporel et le taux de croissance jusqu'à 90 jours d'âge. Le dimorphisme sexuel en faveur des mâles pour la croissance corporelle trouvé dans ce travail est une caractéristique commune exprimée pour les mammifères dans les populations domestiquées. Ce dimorphisme est présent tout au long de la vie des chevreaux mais il augmente proportionnellement de la naissance à l'âge adulte pour atteindre environ $35 \mathrm{~kg}$ chez la femelle et $55 \mathrm{~kg}$ chez le mâle (Najari, 2005).

\section{Distribution des poids aux âges types}

La distribution des poids illustre, outre les potentialités génétiques, les impacts des restrictions environnementales des milieux arides. Les tests appliqués sur les performances des 945 chevreaux ont rejeté l'hypothèse de la normalité pour la plupart des performances, surtout celles réalisées après l'âge d'un mois. Ces résultats confirment que la croissance des chevreaux parait plus conforme aux hypothèses de la génétique quantitative pour les jeunes âges où les phénotypes suivent la forme gaussienne de la distribution. Pour illustrer les effets du milieu aride sur les paramètres statistiques des critères de sélection, il est utile de voir le changement de la distribution des classes de poids en fonction des facteurs du milieu qui affectent le plus les génotypes. Il est bien connu que le facteur année représente une source de variation des performances des animaux élevés sur parcours. Pour cette raison nous avons analysé la nature de la distribution en fonction des années d'étude. La distribution change considérablement d'une année à l'autre pour refléter l'expression des génotypes dans des conditions différentes de pluviométrie. Pendant une année favorable, les performances enregistrées suivent une distribution normale confirmée par les tests de normalité (Kolmogorov et Shapiro-Wilk). Durant l'année 2011 à faible pluviométrie, les poids des chevreaux ont présenté une distribution particulière expliquée par le fait que les chevreaux performants ont été incapables d'extérioriser leur supériorité. Par conséquent, le milieu aride a réduit les performances de la population caprine locale et a limité l'expression de quelques génotypes très productifs.

Cette situation de variabilité mérite d'être approfondie pour contrecarrer ses impacts dans le cadre de l'amélioration génétique de cette population. Une telle distribution obtenue dans des conditions restrictives remet en question la nature réelle du déterminisme génétique des phénotypes. Le principe d'additivité de Falconer (1989), qui considère la performance comme une résultante cumulative des effets des phénotypes et du milieu, est clairement vérifié dans notre cas en raison de l'effet limitant du milieu aride.

\section{CONCLUSION}

Les caprins de la région de Médenine au sud-est de la Tunisie sont de petit gabarit. Leurs performances pondérales sont similaires à celles connues des races locales caprines élevées dans les régions chaudes à ressources restreintes et irrégulières. Le poids faible et le petit format sont considérés comme les conséquences de l'adaptation de la population caprine locale aux conditions de son environnement.

Les performances de croissance des chevreaux ont varié fortement sous les effets de facteurs comme le sexe et l'année de naissance. Les résultats ont souligné l'importance de l'action qualitative du milieu aride sur les phénotypes animaux des candidats à la sélection. En effet, la rareté des ressources et l'irrégularité des conditions environnementales conduisent à des phénotypes qui révèlent des spécificités à prendre en compte par le sélectionneur. Les hypothèses de la génétique quantitative ne sont vérifiées que chez les caprins les plus jeunes et lors d'années favorables. Dans ces cas, les géniteurs disposent de conditions favorables à l'expression de leurs potentialités génétiques ce qui rend possible l'application des procédures de la génétique quantitative. Cependant, lorsque les chevreaux dépassent trois mois d'âge ou lors d'années de disette, 
la base de données des performances parait difficilement ajustable par les approches classiques d'estimation et de prédiction. Dans de telles situations, les géniteurs paraissent, surtout les meilleurs, incapables d'exprimer leurs potentialités génétiques, lesquelles deviennent comparables à celles de caprins ayant des potentialités héritables plus faibles. La conception d'un modèle qui prévoit l'effet inhibiteur de l'environnement sur l'expression des génotypes s'avère dès lors indispensable pour expliquer de pareilles situations. En fait, l'application des méthodologies classiques sans tenir compte de telles spécificités risque de sélectionner contre les animaux les plus performants et les mieux adaptés à la fois.

\section{Conflits d'intérêts}

Les auteurs déclarent que l'étude a été menée sans conflit d'intérêts.

\section{Déclaration des contributions des auteurs}

AA a participé à la conception et à la planification de l'étude, collecté et interprété les données, construit leur analyse, rédigé la première version et les versions successives du manuscrit. MJC a contribué à poser la problématique et participé à la rédaction de l'article. MA a réalisé et organisé les enquêtes de terrain. SN a participé à l'interprétation des données d'enquête, à leur mise en forme et à la rédaction de l'article.

\section{REFERENCES}

Atoui A., Carabaño M.-J., Diaz C., Najari S., 2019. Genetic analysis of live weight of local kids to promote genetic evaluations in the arid areas of Tunisia. Trop. Anim. Health Prod., 1 (10): 1-14, doi: 10.1007/s11250-01902081-z

Dekkers J., 2002. Models for genetic analysis of longitudinal data. University of Guelph, Canada

Falconer D.S., 1989. Introduction to quantitative genetics. Longman, Essex, UK, $438 \mathrm{p}$.

Goffinet B., Elsen J.-M., 1984. Critères optimaux de sélection : quelques résultats généraux. Genet. Sel. Evol., 16 (3): 307-318, doi: 10.1186/1297-968616-3-307

Najari S., 2005. Caractérisation zootechnique et génétique d'une population caprine. Cas de la population caprine locale des régions arides tunisiennes. Thèse Doct., Institut national agronomique, Tunis, Tunisie, 214 p.

Najari S., 2006. Typologie de la chèvre locale dans les régions arides tunisiennes. Institut des régions arides, Médenine, Tunisie, $250 \mathrm{p}$.

Rashidi A., Mokhtari M.-S., Gutiérrez J.-P., 2015. Pedigree analysis and inbreeding effects on early growth traits and greasy fleece weight in Markhoz goats. Small Rum. Res., 124 (10): 1-8, doi: 10.1016/j.smallrumres.2014.12.011

Zhang C., Yang L., Shen Z., 2008. Variance components and genetic parameters for weight and size at birth in the Boer goat. Livest. Sci., 115 (1): 73-79, doi: 10.1016/j.livsci.2007.06.008

\section{Summary}

Atoui A., Carabaño M.J., Abdennebi M., Najari S. Weights of caprine kids of the local Southern Tunisian population under arid conditions: Impacts during selection process

The nature and distribution of quantitative phenotypes represent a basic knowledge for the application of genetic methodologies in domestic animal selection plans. To illustrate the action of non-genetic factors of aridity on the phenotypic distribution of individual growth performance of caprine kids of the local population reared under restrictive and irregular conditions, 945 kids from 285 female goats and 19 male goats, born during the period 1998-2014, were studied by analyzing weight distributions up to 150 days of age. The weight dispersion represented a collective expression of the genotypes of the kids under the study conditions with variations due to both genetic and environmental effects. The assumption of normality was only confirmed at 10 days and 30 days of age when kids were mainly fed under the mother and were not directly dependent on dry land feed resources. In an unfavorable year, the recorded performance followed a particular distribution explained by the fact that performing kids were unable to externalize their superiority. Therefore, the dry land environment reduced the performance of the local goat population. In addition, it limited the expression of some highly productive genotypes. The specificity of the action of the arid environment calls for an in-depth study to adjust the selection methods to the conditions of the restrictive environments and of the adapted genetic groups.

Keywords: goat, livestock systems, growth, selection, environmental factors, Tunisia

\section{Resumen}

Atoui A., Carabaño M.J., Abdennebi M., Najari S. Peso de los cabritos de la población local Sud tunicina en condiciones de aridez: impactos durante procedimientos de selección

La naturaleza y la distribución de los fenotipos cuantitativos representan un conocimiento de base para la aplicación de metodologías genéticas en los programas de selección de los animales domésticos. Con el fin de ilustrar la acción de los factores no genéticos de la aridez sobre la distribución fenotípica de los rendimientos individuales de crecimiento de los cabritos de la población local, criados en condiciones restrictivas e irregulares, 945 cabritos provenientes de 285 cabras y 19 machos cabríos, nacidos durante el período 1998-2014, fueron estudiados mediante el análisis de las distribuciones de pesos hasta 150 días de edad. La dispersión de los pesos representó una expresión colectiva de los genotipos de los cabritos bajo las condiciones del estudio, con variaciones debidas a la vez a los efectos genéticos y ambientales. La hipótesis de la normalidad fue verificada únicamente para las edades de 10 días a 30 días, cuando los cabritos se encontraban sobre todo alimentados bajo la madre y no eran directamente dependientes de los recursos alimenticios del trayecto árido. Durante un año desfavorable, los rendimientos registrados siguieron una distribución particular explicada por el hecho de que los cabritos con mayor rendimiento fueron incapaces de exteriorizar su superioridad. Consecuentemente, el medio árido redujo los rendimientos de la población caprina local. Por otro lado, limitó la expresión de algunos genotipos fuertemente productivos. Esta especificidad de la acción del medio árido incita a una profundización indispensable para ajustar los métodos de selección a las condiciones de los medios restrictivos y de grupos genéticos adaptados.

Palabras clave: caprino, sistemas pecuarios, crecimiento, selección, factores ambientales, Túnez 